\title{
Technè
}

La science au service de l'histoire de l'art et de la préservation des biens culturels

$44 \mid 2016$

Archives de l'humanité : les restes humains patrimonialisés

\section{Les collections de peaux humaines tatouées}

The collections of tattooed human skins

\section{Éloïse Quétel}

\section{(2) OpenEdition}

1 Journals

\section{Édition électronique}

URL : http://journals.openedition.org/techne/1186

DOI : 10.4000/techne. 1186

ISSN : 2534-5168

Éditeur

C2RMF

\section{Édition imprimée}

Date de publication : 1 novembre 2016

Pagination : 114-117

ISBN : 978-2-7118-6339-6

ISSN : 1254-7867

Référence électronique

Éloïse Quétel, « Les collections de peaux humaines tatouées », Technè [En ligne], 44 | 2016, mis en ligne le 19 décembre 2019, consulté le 25 juillet 2020. URL : http://journals.openedition.org/techne/ 1186 ; DOI : https://doi.org/10.4000/techne.1186

\section{(c) $\oplus \Theta \Theta$}

La revue Technè. La science au service de l'histoire de l'art et de la préservation des biens culturels est mise à disposition selon les termes de la Licence Creative Commons Attribution - Pas d'Utilisation Commerciale - Pas de Modification 4.0 International. 

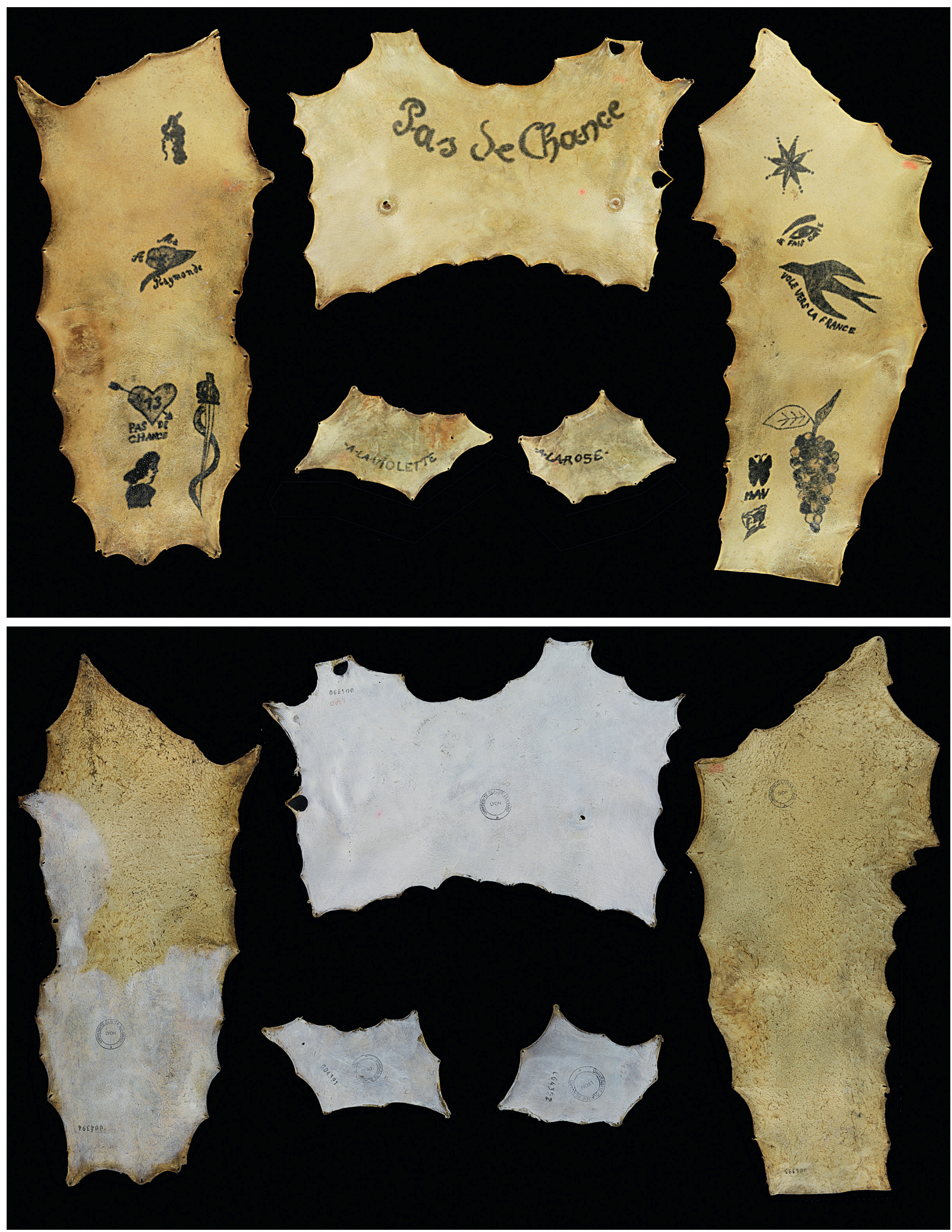

Fig. 1 et 2. Les cinq fragments de peaux humaines tatouées de la collection du musée d'Anatomie Testut-Latarjet, face et revers. @ É. Quétel. 


\section{Éloïse Quétel \\ Les collections de peaux humaines tatouées}

The collections of tattooed human skins

Résumé. Prélevées entre la fin du XIX $X^{e}$ siècle et le milieu $d u X X^{e}$ siècle, les peaux humaines tatouées étaient conservées comme support d'étude dans le domaine de l'anthropologie et de la médecine, à la fois pour leurs aspects criminologiques, mais aussi pour leurs aspects esthétiques.

Cinq fragments de peau issus d'un même corps, auparavant conservés au sein du musée d'Anatomie Testut-Latarjet de Lyon ${ }^{1}$, ont été choisis afin de réaliser une étude portant sur les différentes problématiques liées à leur statut de restes humains conservés dans les institutions muséales.

Mots-clés. Peaux humaines tatouées, cadavre, pathologie, anthropologie criminelle, conservation-restauration, déontologie.
Abstract. Removed from their bodies between the late-19th and mid-20th century, tattooed human skins were kept as a basis for study in the fields of anthropology and medicine, both for their criminological and aesthetic aspects. Five fragments of skin from the same body, previously conserved in the Testut-Latarjet Anatomy Museum, Lyon, were selected in order to carry out a study on the different problems related to their status as human remains present in museum collections.

Keywords. Tattooed human skins, corpse, pathology, criminal anthropology, conservation-restoration, deontology.

\section{Les collections de peaux humaines tatouées}

Les collections de peaux humaines tatouées font partie d'un patrimoine caché, dit " patrimoine noir» de l'humanité 2 . Le plus souvent oubliées au sein des institutions les conservant et/ou volontairement mises de côté à cause de leur statut ambivalent, elles suscitent néanmoins des questions éthiques et déontologiques spécifiques appliquées aux restes humains, ainsi que des considérations particulières concernant leur conservation.

Le prélèvement de peaux humaines tatouées a été majoritairement réalisé entre la fin du XIX ${ }^{\mathrm{e}}$ et le début du XX ${ }^{\mathrm{e}}$ siècle (et de manière isolée, lors de la Seconde Guerre mondiale). Habituellement prélevées après le décès ${ }^{3}$, pour identifier un individu, ou conserver ses tatouages de manière pérenne (études criminologiques et esthétiques), la peau était découpée au plus près du dessin, tannée et tendue afin de la faire sécher. Néanmoins, même si l'on possède quelques rares traces et écrits de collectionneurs ou médecins les ayant collectés ${ }^{4}$, on constate une absence d'informations précises quant à ces prélèvements.

Ces collections sont conservées au sein d'institutions variées, tels que le Muséum national d'histoire naturelle, les facultés de médecine, les instituts médico-légaux, les musées de médecine, de pathologie, de pharmacie et des sciences. Dans le but de se prémunir des réactions «épidermiques » provoquées par leur historicité (criminalité, expériences médicales douteuses ${ }^{5}$ ), ces collections demeurent méconnues du public et restent le plus souvent cantonnées au sein des espaces de réserves.

\section{La collection du musée d'Anatomie Testut-Latarjet}

Le musée d'Anatomie Testut-Latarjet de Lyon était consacré à l'anthropologie criminelle, l'anatomie générale et comparée, la tératologie, la parasitologie, etc., et conservait une collection de peaux humaines tatouées, constituée d'une quinzaine de pièces conservées sèches ou en fluide (en solution formolée).

$\mathrm{Au} \mathrm{XIX}^{\mathrm{e}}$ siècle, le tatouage est un signe pathognomonique de la criminalité et représente une dégénérescence de la société. Il sera le fer de lance des criminologues, tel Alexandre Lacassagne, qui, dans le cadre de ses recherches sur l'identification du criminel, observera et collectera plus de deux mille tatouages sur cent cinquante individus, à la fois en réalisant des calques à même les corps des tatoués, mais aussi en réalisant des prélèvements sur les cadavres. Ses études portaient notamment sur l'emplacement, la diversité, la signification et la récurrence des différents motifs des tatouages observés. Dans ce cadre, il constitua la collection de peaux humaines tatouées du musée. 
Afin de réaliser cette étude, nous avons sélectionné cinq peaux au sein de la collection : la peau d'un torse, de deux bras et de deux dessus de pied (fig. 1 et 2). Ces dernières ont été choisies pour leur singularité, dans un premier temps car la forme de leur découpe correspondait à la forme de la zone corporelle prélevée, mais aussi parce que ces cinq peaux provenaient d'un seul et même individu, ce qui est encore plus rare. En effet, les fragments de peaux étaient le plus souvent prélevés et découpés en suivant les contours du (ou des) 116 tatouage(s), afin de réaliser un simple catalogue iconographique. Ici, la forme donnée à ces prélèvements prouve que l'intention était de conserver un corps, donc un individu, au-delà de l'esthétique de ses tatouages.

Il nous a fallu glaner des informations au fur et à mesure de nos recherches afin de découvrir l'existence d'autres collections conservées à travers le monde ${ }^{6}$. Cela nous a permis d'identifier plusieurs types de préparations, d'études et de méthode de conservation, notamment le tannage ou la conservation en fluide avec une solution à base de formaldéhyde, d'allantoïne ou d'alcool.

\section{La conservation et la restauration}

Suite à l'observation de la collection lyonnaise, nous avons constaté que les altérations étaient parfois similaires à celles constatées sur les autres collections de peaux humaines tatouées et tannées, notamment l'empoussièrement, l'encrassement, des fissures, des cassures et des efflorescences graisseuses.

Concernant les cinq peaux de la collection lyonnaise, les altérations provenaient d'un stockage inadapté (les différentes peaux de la collection étaient empilées les unes sur les autres au fond d'une vitrine, sans interfaces ni conditionnements particuliers), générant ainsi des contraintes de manipulation, un empoussièrement et un encrassement. L'apparition de taches, les efflorescences cristallines et les remontées graisseuses dues à l'application d'huile d'olive et de cire blanche au revers des peaux relèvent des particularités liées à la mise en œuvre ${ }^{7}$ (fig. 2). Par ailleurs, le manque total d'informations concernant ces cinq peaux fut également problématique. Appelée dissociation ${ }^{8}$, cette altération métaphysique est néanmoins importante puisque le sujet perd son contexte et sa valeur informationnelle.
Fig. 3. Les cinq fragments de peaux humaines tatouées dans leur nouveau conditionnement en polypropylène avec planches de consultation recouvertes de plastazote, cales en Ethafoam et système de maintien en fil d'acier inoxydable gainé de Téflon (PTFE). (C) É Quétel.

Les étapes de conservation et de restauration ont ainsi consisté en un dépoussiérage à l'aide d'un aspirateur à filtre HEPA et d'une petite brosse souple, d'un léger nettoyage à la salive pour les zones encrassées, et en la réalisation de recherches approfondies afin de recontextualiser la collection dans son ensemble. Le conditionnement et les planches de consultation ont été réalisées avec du polypropylène cannelé et ces dernières ont été recouvertes de mousse de polyéthylène. De plus, des tiges en fil d'acier inoxydable amovibles, gainés de polytétrafluoroéthylène ont été disposés sur le pourtour de chaque peau afin de les stabiliser (fig. 3).

\section{Monstration et reconnaissance patrimoniale}

Observer des peaux humaines tatouées suscite chez le visiteur une réflexion sur lui-même et sur son propre corps. La peau humaine est une couche perméable et imperméable qui enrobe tout notre corps et crée une barrière avec l'environnement extérieur, mais elle est aussi le véhicule de nos émotions et de notre personnalité. Lors des autopsies, on cache le visage 
du cadavre, et même parfois ses tatouages afin de ne pas provoquer de mal-être. On peut donc aisément comprendre que le visiteur puisse être plus facilement choqué à la vue d'une peau humaine que d'un squelette. Par ailleurs, compte tenu de la recrudescence du phénomène du tatouage depuis cette dernière décennie et face à un public contemporain, le transfert est encore plus évident. La peau tatouée met alors en exergue une personnalité, un individu à qui l'observateur peut s'identifier.

Conserver et exposer des corps ou des morceaux de corps implique une réflexion quant à leur place et leur valeur au sein de notre société, mais aussi, à un apprentissage de respect, et d'attitudes réfléchies et adaptées concernant leur matérialité (ICOM, articles 2.5, 3.7 et 4.3 sur la question des matériaux culturels sensibles). C'est pourquoi une réflexion est menée lors de l'exposition de restes humains, ainsi que dans le cadre de la gestion au quotidien de ce type de spécimen.
Les cinq peaux humaines tatouées de Lyon sont entrées au sein de la collection du musée d'Anatomie Testut-Latarjet suite à la découverte du corps dans l'un des congélateurs du laboratoire d'anatomie. La dépouille n'ayant jamais été réclamée par sa famille, les peaux une fois prélevées sont directement passées du processus de «néantisation " (le corps a perdu son identité au profit de sa seule dimension matérielle) à celui de leur patrimonialisation, néanmoins, sans prise en charge particulière concernant leur conservation.

Bien souvent, la conservation et la restauration des restes humains demeurent anecdotiques. Si l'on constate aujourd'hui un regain d'intérêt pour les peaux humaines tatouées (on citera par exemple la récente exposition « Tatoueurs, Tatoués » au musée du quai Branly), la question demeure entière de savoir si cela peut déboucher, à terme, sur une véritable considération de ces collections, assortie de mesures plus régulières quant à leur conservation et leur restauration.

\section{Notes}

1. Le musée d'Anatomie Testut-Latarjet a été fermé en 2015. Les collections rejoindront bientôt le futur "musée des Sciences Médicales et de la Santé » à Rillieux-la-Pape.

2. "Cet immense ensemble d'objets et d'archives mais aussi de bâtiments qui donnent à voir une facette moins glorieuse de notre histoire ", Artières, Salle, 2009, p. 158 .

3. Parfois, la peau était prélevée sur le vivant, l'infection du tatouage dû à l'utilisation de pigments nocifs et le danger d'une septicémie motivaient le prélèvement.

4. Notamment, Alexandre Lacassagne, professeur de jurisprudence et médecin légiste, qui a constitué la collection du musée Testut-Latarjet de Lyon (Lacassagne, 1881), le Dr Masaichi Fukushi, physicien et pathologiste, qui a constitué la collection de l'université de Tokyo (Life and Death tattoos, magazine Tattootime $\mathrm{n}^{\circ} 4,1989$, éditions Hardy marks), et le docteur Rudolf Virchow, pathologiste qui a constitué une partie de la collection du Museum Der Charité de Berlin (Tatouages dans les musées de pathologies, Denis Durand de Bousingen, Tatouage magazine $n^{\circ} 58,2007$, éditions Larivière).

5. L'anthropologie criminelle se basait sur des critères d'évaluation physiques et désuets et discriminants. Lors de la Seconde Guerre mondiale, au camp de Buchenwald, les prisonniers tatoués étaient écorchés (The Beasts of Buchenwald, Karl and Ilse Koch, Human skin lampshades, and the war-crimes trial of the century, Flint Whitlock, Cable Publishing, Brule, Wisconsin, 2011). 6. Notamment au département de pathologie de l'université de médecine de Tokyo, au Pathologisch-anatomische Bundesmuseum (ou Narrenturm) de Vienne, au Medizinhistorisches Museum der Charité de Berlin, à la Wellcome collection du Science Museum de Londres, au conservatoire d'anatomie de Montpellier, au musée de l'Homme de Paris, au Muséum d'histoire naturelle de Rouen, au Museum of Health and Medicine de Washington. 7. Les peaux ont été fixées avec une solution à base de formaldéhyde, puis tendues sur un cadre en bois. Une couche de cire blanche a été déposée au revers afin d'opacifier la couleur des peaux et de l'huile d'olive a été appliquée sur la face afin de faire ressortir le pigment noir des tatouages. 8. R. Robert Waller et Paisley S. Cato, ICC, Conservation préventive et risques, agents de détériorations. http://canada.pch. gc.ca/fra/1444924574622\#def1.

\section{Bibliographie}

Andrieux J.-Y., article de Frangne P.H, Patrimoine, sources et paradoxes de l'identité, Actes du cycle de conférences prononcées à l'Université Rennes 2, 5 novembre 2007-2 avril 2008, coll. "Art et société », Presses universitaires de Rennes, 2011
Artières P., Salle M., 2009, Papiers des bas fonds, archives d'un savant du crime, 1843-1924, éditions Enquête d'archives, Paris.

Cadot L., 2009, En chair et en os : le cadavre au musée. Valeurs, statuts et enjeux de la conservation des dépouilles humaines patrimonialisées, École du Louvre/RMN, Paris.

Cassman V., Odegaard N., Powell J., 2006, Human Remains, Guide for Museum and Academic Institutions, Altamira press, U.S.

Code de déontologie de l'ICOM pour les musées, 2013.

Code de la santé publique, Article L1232-1, dites Loi Bioéthiques 1994, modifié par la loi $\mathrm{N}^{\circ} 2004-800$ du 6 août 2004

Cornu M., 2009, Le corps humain au musée, de la personne à la chose?, Recueil Dalloz.

Lacassagne A., 1881, Les tatouages : étude anthropologique et médico-légale, éditions Baillière, Paris

Quétel E., 2013, Les peaux humaines tatouées, de l'anthropologie criminelle à l'intention esthétique, mémoire de fin d'études, ESAA, Avignon.

Symposium du musée du quai Branly, 2008, «Des collections anatomiques aux objets de culte : conservation et exposition des restes humains dans les musées [en ligne] ", Actes du colloque, musée du quai Branly, 22-23 février 2008. http://www.quaibranly.fr/ 